\title{
Subjecting Radiologic Imaging to the Linear No-Threshold Hypothesis: A Non Sequitur of Non-Trivial Proportion
}

\author{
Jeffry A. Siegel ${ }^{1}$, Charles W. Pennington ${ }^{2}$, and Bill Sacks ${ }^{3}$ \\ ${ }^{1}$ Nuclear Physics Enterprises, Marlton, New Jersey; ${ }^{2}$ NAC International (retired), Norcross, Georgia, and executive nuclear energy \\ consultant, Alpharetta, Georgia; and ${ }^{3}$ U.S. Food and Drug Administration (retired), Green Valley, Arizona
}

Radiologic imaging is claimed to carry an iatrogenic risk of cancer, based on an uninformed commitment to the 70-y-old linear nothreshold hypothesis (LNTH). Credible evidence of imaging-related low-dose ( $<100 \mathrm{mGy}$ ) carcinogenic risk is nonexistent; it is a hypothetical risk derived from the demonstrably false LNTH. On the contrary, low-dose radiation does not cause, but more likely helps prevent, cancer. The LNTH and its offspring, ALARA (as low as reasonably achievable), are fatally flawed, focusing only on molecular damage while ignoring protective, organismal biologic responses. Although some grant the absence of low-dose harm, they nevertheless advocate the "prudence" of dose optimization (i.e., using ALARA doses); but this is a radiophobia-centered, not scientific, approach. Medical imaging studies achieve a diagnostic purpose and should be governed by the highest science-based principles and policies. The LNTH is an invalidated hypothesis, and its use, in the form of ALARA dosing, is responsible for misguided concerns promoting radiophobia, leading to actual risks far greater than the hypothetical carcinogenic risk purportedly avoided. Further, the myriad benefits of imaging are ignored. The present work calls for ending the radiophobia caused by those asserting the need for dose optimization in imaging: the low-dose radiation of medical imaging has no documented pathway to harm, whereas the LNTH and ALARA most assuredly do.

Key Words: radiological imaging; linear no-threshold; ALARA; hormesis; adaptive response; radiophobia

J Nucl Med 2017; 58:1-6

DOI: 10.2967/jnumed.116.180182

$\mathbf{T}$ he linear no-threshold hypothesis (LNTH) has been applied to low-dose and low-dose-rate ionizing radiation for more than $70 \mathrm{y}$ but, lacking valid scientific foundation, remains a hypothesis. Nonetheless, this hypothesis is the orthodox foundation of radiation protection science, in turn forming the basis of regulations and public policy.

The LNTH derives from incomplete, early-20th-century geneticexperimental observations yielding inaccurate conclusions, undetected by other scientists until quite recently (1). Hermann Muller, in his 1946 Nobel Lecture, asserted that a no-harm threshold was nonexistent, since linearity had been demonstrated for doses down to

Received Jun. 24, 2016; revision accepted Jul. 18, 2016.

For correspondence contact: Jeffry A. Siegel, Nuclear Physics Enterprises, 4 Wedgewood Dr., Marlton, NJ 08053.

E-mail: nukephysics@comcast.net

Published online Aug. 4, 2016.

COPYRIGHT (C 2017 by the Society of Nuclear Medicine and Molecular Imaging.
4,000 mGy, a stunning non sequitur. Nor has any evidence since validated the carcinogenicity of low doses. The LNTH extrapolation from evidence-supported, high-dose effects to putative lowdose responses claims that all acute ionizing radiation exposure down to zero is harmful proportionally to dose and that it yields cumulative harm throughout life, regardless of how low the dose rate. Both claims are demonstrably false and harmful, leading to LNTH-derived regulations and policies that are not protective $(2,3)$; for example, more than 1,600 deaths resulted from the LNTH-based evacuation policy for nearby residents after the Fukushima nuclear accident (3).

Throughout time, we have been bathed in low-dose radiation from land, sky, and our own bodies. Today's average annual natural background exposure ranges from 1 to $260 \mathrm{mSv}$ in some places on the planet. No associated adverse health effects have been documented anywhere (4). For comparison, typical CT and combined whole-body ${ }^{18}$ F-FDG PET/CT scan doses delivered acutely are 10 and $14 \mathrm{mSv}$, respectively. This radiation-rich history implies that extant life-forms must have developed adaptive, biologic repair and/ or removal responses to radiation damage. The primary LNTH fallacy is it excludes this evolutionary biology, ignoring the body's differing responses to high versus low radiation doses (5). Low doses stimulate protective responses; high doses overwhelm and inhibit such protections.

Herein are offered dissenting views on subjecting medical imaging to the LNTH, as this hypothesis is characterized by its one-sided failure to incorporate experimental research findings and its support by erroneous mathematic and statistical maneuvers that merely confirm a priori assumptions through circular reasoning. Our heterodoxy maintains that this one-sidedness is twofold: first, it focuses on unquestioned radiogenic cellular damage while ignoring the organism's proven biologic responses to mitigate that damage plus the endogenous damage (due to normal metabolism) several orders of magnitude greater; second, it focuses on only the hypothetical risks of imaging while ignoring its myriad benefits and the actual risks associated with its alternatives (6-8). The hypothetical risks of medical imaging pale in comparison to these actual risks.

Unwarranted fear of low-dose radiation leads to the misguided doctrine of "prudence" in dosing-ALARA (as low as reasonably achievable) — that, by often diminishing image quality, increasingly produces suboptimal and even nondiagnostic CT scans $(9,10)$. Thus, today, $70 \mathrm{y}$ after Muller's Nobel speech, another non sequitur advances, this time within the field of radiologic imaging. This article provides a scientific rebuttal of the key errors within the LNTH orthodoxy to rehabilitate and restore low-dose radiation's position of respect within science and medicine and to help undo needless public and professional radiophobia. 


\section{FAILURE OF THE LNTH GOLD STANDARD}

The atomic-bomb survivor cohort of the Life Span Study (LSS) is the single most important dataset-the gold standard-for estimating radiation effects in humans $(5,11)$. The 1958-1998 LSS data for acute exposure to low-dose, low linear-energy-transfer radiation, such as the $\mathrm{x}$ - and $\gamma$-rays used in medical imaging, were reported by the Biologic Effects of Ionizing Radiation (BEIR) VII Committee in 2006 (11) to be consistent with the LNTH doseresponse relationship for development of solid cancers. The BEIR Committee operates under the auspices of the National Academy of Sciences, receiving significant financial support from various regulatory and other government agencies; thousands of government and private industry jobs depend on the conclusions of the BEIR Report, which promotes acceptance of the LNTH. The 2005 French Academy of Sciences Report (12), however, reached very different conclusions. Providing evidence for protective adaptive responses and finding no valid evidence for harm below $100 \mathrm{mGy}$, the report questioned the validity of the LNTH in that range.

The BEIR VII Committee, seeking putative low-dose cancer-risk $(<100 \mathrm{mGy})$ reductions but unwilling to forgo linearity, introduced the artifice of the "dose and dose-rate effectiveness factor." A factor of 1.5 was chosen to reduce the slope of the LNTH-derived result, though the LSS data are not linear at doses lower than $100 \mathrm{mGy}$ $(3,13)$. Rather, linearity is forced by the linear no-threshold model from high-dose extrapolation. Independent analyses of LSS data indicate an apparent threshold as high as $55 \mathrm{mGy}$, comparable to a threshold of $60 \mathrm{mSv}$ reported by others (3).

Using updated LSS data, Ozasa et al. (14) reported that 0-180 mGy was the lowest dose range exhibiting no significant, acute-dose, excess relative risk (ERR) for all-solid-cancer mortality. In this dose range, the ERR uncertainty (from their Fig. 4) overwhelms its dose dependence, with the $95 \%$ confidence intervals including negative ERR values, consistent with a beneficial, as well as a harmful, effect. This uncertainty is not reflected in the linear dose response or its confidence range because that linear fit was estimated by extrapolation from dose levels of 1 Gy or higher (15).

Ozasa et al. used Poisson regression methods to mathematically derive background mortality rates at zero dose, which effectively enables the lowest-dose cohorts to determine this rate by linear extrapolation to zero dose. Other studies show reduced mortality rates in low-dose cohorts (16), compared with cohorts experiencing no radiation above natural background; therefore, Poisson regression introduces negative bias in the background mortality rate, which artificially elevates the reported ERR values.

Correcting this bias, ERR values become negative for doses below approximately $0.6 \mathrm{~Gy}$, beneficially reducing cancer risk relative to background cancer rates (16). Another LSS reanalysis (17) exhibits negative ERRs below a threshold at $200 \mathrm{mSv}$, again consistent with radiation-induced benefit.

\section{CONTRARY TO THE LNTH, BIOLOGY RESPONDS ADAPTIVELY}

The LSS data do not support the LNTH; rather, the observed thresholds and negative ERRs agree with experimental evidence for adaptive cancer protection after low-dose radiation exposure. These data are more consistent with a radiation hormetic (protective) model than with the linear no-threshold (harm at any dose) model. Yet, John Boice, president of the National Council on Radiation Protection and Measurements, continues to assert that the LNTH is the most plausible hypothesis (18) (this council is a congressionally chartered, private corporation that receives financial support from federal radiation regulators and other governmental agencies).

Whether or not low-dose damage is linear, the body's defensive response is nonlinear, leaving the net result nonlinear (19). The body deals with this damage through a set of proven mechanisms, collectively called the adaptive response $(3,20,21)$, which offers cancer protection through DNA repair involving more than 150 genes, antioxidant production, apoptosis on the cellular level, bystander effects on the tissue level, and immune-system removal of surviving damaged cells on the organismal level. Double-strand-break repair occurs even after low-dose CT scans (22). Numerous studies demonstrate at least 6 mechanisms for reducing cancer rates and increasing longevity, stimulated by low-dose damage (23).

BEIR VII (11) grants the existence of "incomplete" repair, but because imperfect repair of initial DNA damage is assumed, the BEIR Committee dismisses a low-dose threshold for carcinogenicity, ignoring additional mechanisms of defense against radiationcaused damage when DNA repair fails. The report cites a paper by Rothkamm and Löbrich (24) but proceeds to misrepresent their findings (23). The paper provides evidence for mechanisms reducing both spontaneous and radiation-induced damage below spontaneous levels (a hormetic effect), by directly measuring the progression of doublestrand-break foci at low doses. Postirradiation counts of cultured cells with double-strand breaks were found to decrease to preirradiation counts, constituting evidence of repair or cell-destroying apoptosis-a finding not mentioned in the BEIR VII report.

The LNTH asserts that radiation damage is cumulative, no matter the dose or dose rate. But this is directly contradicted by the practice of fractionation of high-dose radiation therapy, demonstrating that recovery occurs between treatments (25). More importantly, because low doses stimulate repair or removal of radiogenic damage in excess of that immediate damage, they provide enhanced protections against additional damage over time, including damage from subsequent higher radiation exposures, infections, endogenous production of reactive oxygen species, and other nonradiogenic damage. The net result is reduction of damage below spontaneous levels $(21,26)$, likely contributing to a lifetime-cancer-risk reduction.

Further, spontaneous levels of DNA alteration resulting from a cell's normal metabolic processes dwarf those due to low-dose radiation $(3,27)$. For example, the average annual U.S. background of $3 \mathrm{mSv}$ produces 3-30 DNA alterations per cell per year, and an acute-dose CT scan about 10-100 alterations per cell, whereas mutation rates due to the body's normal metabolic chemistry are a million times higher. Thus, the LNTH extrapolation of high-dose levels (which are inhibitory of protective mechanisms) down to low-dose levels falsely predicts detrimental effects at a low dose.

Another study, involving radiation exposures to interventional cardiologists (median of $4 \mathrm{mSv} / \mathrm{y}$ ), compared them with unexposed controls. Low-dose, chronic exposure was associated with two adaptive cellular responses: enhanced antioxidant defense and increased apoptotic response (28). These likely compensate for increased reactive oxygen species production and contribute to maintaining cellular homeostasis. An accompanying editorial noted that these data confirm low-dose protective responses (29).

Mutations are necessary, but not sufficient, to produce clinically overt cancer. The immune system generally keeps cancers in check, and cancers develop mainly when the immune system is suppressed. The role of the immune system in cancer development now replaces the outdated "one mutation = one cancer" model. Recent research shows the inaccuracy in mechanistic models of radiation-induced cancer suggesting that double-strand breaks 
lead to chromosome aberrations resulting in cancer. Low-dose radiation has been shown to stimulate the immune system, causing a reduction in cancer rates (30). Furthermore, residents in areas with a higher background radiation level $(3.3 \mathrm{mSv} / \mathrm{y})$ were found to have higher frequencies of chromosome aberrations than lowerbackground control populations $(1.1 \mathrm{mSv} / \mathrm{y})$ yet had lower all-cancer mortality, indicating that the frequency of chromosomal aberrations may not be suitable as a surrogate for cancer mortality (31).

The evidence for the biologic-response/cancer-reduction paradigm of low-dose radiation continues to mount. The 2015 Nobel Prize in Chemistry was awarded for research by Tomas Lindahl, Paul Modrich, and Aziz Sancar showing how cells safeguard genetic information, preventing it from disintegrating into chaos, through a host of molecular systems that continuously monitor and repair DNA.

\section{ABSENCE OF ACUTE, LOW-DOSE RADIATION CARCINOGENESIS IS EVIDENCE}

As previously noted with the revised Ozasa et al. (14) data, most ERRs in the low-dose range have confidence intervals that include negative values. Negative values suggest that imaging doses reduce rather than increase cancer risk compared with a valid baseline.

The most widely used estimate for the slope of the radiationinduced cancer-mortality dose-response relationship is about $5 \%$ per gray for an all-age population. This estimate is primarily derived from LSS data at 1 Gy or higher using a linearity-preserving artifice, the dose and dose-rate effectiveness factor, with a value of $2(15,32)$, and from the added nonempiric assumption that there is no threshold. This may be verified by a point-estimate calculation at $1 \mathrm{~Gy}$ (i.e., $5 \%$ at $1 \mathrm{~Gy}$ ) based on the LSS data (14), but it is not a valid predictor of risk at lower doses; for example, a 10-mGy CT dose would represent a hypothetical $0.05 \%$ risk estimate (corresponding to an ERR of 0.004). As can be seen from these same data, below about $200 \mathrm{mGy}$ the dose-response relationship is not an extrapolated line from higher doses but instead is roughly horizontal. Therefore, both the "L" and the "NT" components of "LNT" are false. Since LNTH-derived, low-dose-risk estimates have huge uncertainties and are not validated by observed LSS data (from which they are derived), these risk estimates are not merely notional but flatly false.

Advocates excuse their inability to provide low-dose and lowdose-rate evidence for the LNTH, claiming this inability is because the ratio of radiogenic low-dose cancer risk (the "signal") to the variation in spontaneous cancer risk (the "noise") is too small to distinguish signal from noise. This explanation for radiogenic signal invisibility is a red herring for radiologic imaging, including for children irradiated by up to $200 \mathrm{mSv}$. Solid-cancer incidence rates among the Hiroshima and Nagasaki atomic bomb survivors who were younger than $6 \mathrm{y}$ when the bombings occurred were examined by Preston et al. (33). Their reported relative risk values and our analyses of the raw data in their Table 3 indicate no significant difference between the adult-onset solid-cancer incidence of the control group and that of subjects who had received exposures of up to $200 \mathrm{mSv}$ as children; this agrees with Ozasa's adult results (14) and therefore suggests that children are not more radiosensitive to harmful effects at low doses.

Hundreds of studies have demonstrated the health benefits of low-dose radiation exposure, and not just absence of harm. These include, for example, reduced cancer incidence and reduced allcause mortality, that is, increased longevity $(12,21,23)$. Since these demonstrated benefits, unlike the LNTH-predicted harms, are not rendered invisible by noise, LNTH advocates simply ignore or distort the evidence for benefit.

Nonetheless, these studies show radiogenic cancer-signal invisibility has some validity, but for a different reason. Adaptive responses likely negate a significant portion of the radiogenic signal, forcing the signal-to-noise ratio toward zero. But science cannot observe pure radiogenic signals separately from spontaneous cancer noise because they may be inseparable. According to Ozasa (15) it is difficult to estimate radiogenic risk at low doses because acute A-bomb low doses must be calculated on top of an uncertain background dose and these two values can overlap, becoming indistinguishable.

Although this discussion applies to acute doses (such as CT imaging), the total dose from nuclear medicine procedures is protracted, which is known to reduce risk compared with acute exposure of the same total dose (34). Studies involving thousands of children younger than $20 \mathrm{y}$ who received ${ }^{131} \mathrm{I}$ for diagnostic purposes ( $<3.7 \mathrm{MBq}$; small children, $<0.37 \mathrm{MBq}$ ) have been reported by Siegel and Silberstein (35). These children, some followed for $40 \mathrm{y}$, received mean thyroid doses of about $1 \mathrm{~Gy}$. No evidence of increased risk of thyroid cancer due to childhood intake of ${ }^{131} \mathrm{I}$ was found.

\section{EVALUATION OF THE PRESENTED EVIDENCE}

The evidence presented shows a reduced, not increased, cancer risk at radiologic imaging doses, and the LSS data show the LNTH-predicted, low-dose carcinogenicity is invalid up to approximately $200 \mathrm{mGy}$. Thus, the much lower doses from medical imaging of children and adults should not be feared or avoided for radiophobic reasons. A typical CT scan effective dose is about $10 \mathrm{mSv}$; a PET/CT brain scan, 5-7 mSv; and a routine whole-body ${ }^{18}$ F-FDG PET/CT scan, $12-15 \mathrm{mSv}$ (36). In general, epidemiologic studies that focus on providing direct low-dose LNTH-consistent risk estimates fail to address the basic sciences (e.g., biology and chemistry) and use often-hidden circular reasoning (assuming that which must be demonstrated empirically), thereby rendering their conclusions false and indefensible (23).

Although recent large epidemiologic studies-Pearce et al. (37) and Mathews et al. (38) - suggested an increased low-dose cancer risk associated with pediatric CT scans, these results have been effectively rebutted. Major flaws are their willingness to draw causal conclusions from mere association and their failure to consider that the association is likely due to reverse causation (i.e., cancer or illness gives rise to CT, not the reverse). Additionally, inaccurate dosimetry and implausible risk estimates are apparent. Other recent large-scaled cohort studies examining pediatric CT cancer risk-Journy et al. (39) and Krille et al. (40)—concluded that confounding by indication and reverse causation must be ruled out completely, or observed excess cancer risk may be falsely, and facilely, attributed to CT exposure.

Radiologic imaging, nuclear medicine procedures, and, therefore, cumulative public radiation doses have increased dramatically over several decades, but their contributions to reduced morbidity and improved longevity have also increased. Concomitantly, concerns have arisen that radiation produces a higher radiogenic cancer risk. The dose-optimization movement of pediatric imaging led to the "Image Gently" campaign, which seeks to lower doses. Consensus guidelines for administered activity for pediatric nuclear medicine studies have been developed (41) that advise lower doses, based on the LNTH: "A reasonable assumption is to apply the linear no-threshold hypothesis for radiation-induced carcinogenesis when making judgments about the relative radiation-associated risks of different imaging studies" 
(42). Yet, as demonstrated herein, assuming the LNTH accurately assesses risk in the face of voluminous evidence to the contrary can never be called "reasonable."

The usual justification for this assumption is that it errs on the side of caution - the precautionary principle, which may be useful if action to control the feared agent has no, or less harmful, side effects. However, for radiologic imaging, significant collateral negative consequences of lowering dose arise. Reducing patient doses to mitigate purely hypothetical cancer risks increases other well-known risks resulting from fear of imaging (7). These include imaging avoidance, nondiagnostic image quality, and use of alternative imaging procedures, such as a longer-duration MRI study, requiring risk-incurring sedation for young children (6-8). The risks of misdiagnoses from inadequate dose could be much higher than the cancer risks that the LNTH falsely predicts and that are putatively avoided by ALARAbased dose-reduction strategies (9).

\section{A NON SEQUITUR: MEDICAL IMAGING SHOULD BE INFLUENCED BY THE LNTH}

Discussing potential risks and ignoring corresponding benefits is improper and even harmful (43); unfortunately, quantitative estimates demonstrating relative and absolute benefits of diagnostic imaging are uncommon $(6,8)$. Further, comparing long-term cancer risks with the present benefit from an imaging study is not a liketo-like comparison. For example, in a CT study in young adults, underlying medical morbidity, rather than CT-induced cancer, was shown as the much greater driver of adverse patient outcomes; the observed risk of a patient dying within $5 \mathrm{y}$ from the underlying disease was at least 1-2 orders of magnitude greater than the hypothetical LNTH-derived risk of dying from CT-induced cancer (44).

The very concept of dose "optimization" (ALARA dosing), is onesided and therefore flawed, ignoring much greater, fear-driven risks, along with the likely dual benefits of imaging: first, the diagnostic information provided, including more accurate and rapid diagnoses, lives saved, quality-of-life improvements, reduced hospital stays, and cost reduction (8) (e.g., we know that $\mathrm{CT}$ scans strengthen confidence in prior diagnoses, often leading to better treatments or more accurate diagnoses (45)); and second, the far more likely lifetimecancer-risk reduction resulting from the radiation itself $(21,23,26,30)$.

A recent study demonstrated a substantial benefit from the use of PET/CT scans to assess response to chemoradiotherapy for primary treatment of patients with squamous-cell head and neck carcinoma with advanced nodal disease (46). The trial assessed the noninferiority of PET/CT-guided surveillance of planned neck dissection performed only if imaging showed an incomplete or equivocal response. The primary endpoint was overall survival. Survival was similar between patients undergoing PET/CT-guided surveillance and patients undergoing neck dissection, but surveillance resulted in considerably fewer operations ( $\sim 80 \%$ of patients avoided neck dissection), which was additionally more cost-effective. Dissection is generally a 3 -h operation, involving both considerable morbidity and potentially long hospital stays. Early and accurate diagnosis through medical imaging reduces mortality, the need for treatment, and costs.

Brenner et al. (47) perhaps started the frenzy over CT dose and cancer risk. On the basis of an LNTH calculation involving unsupported parameters with significant uncertainties, they projected that approximately 500 children under the age of $15 \mathrm{y}$ would die of cancer attributable to CT radiation. The irresponsibility of this projection was underscored by International Commission on
Radiological Protection Publication 103 (48) and others stressing that the low-dose risk uncertainties of the LNTH show it should not be used to calculate hypothetical cancers from small radiation doses received by large populations. According to Lauriston Taylor (25), this type of calculation is based on a literal application of the LNTH, treating it as fact even though there is no statistical or other verification of this calculation. Such claims, he said, are "deeply immoral uses of our scientific knowledge." Estimating future CT-caused cancers on the basis of the unsupportable assumptions of the linear no-threshold model (e.g., the fallacious $5 \% / \mathrm{Sv}$ cancer risk) results in a purely fictitious prediction serving only to generate fear-based negative consequences.

The goal of dose management should be aimed at achieving diagnostic-quality images, not reducing dose in the hormetic imaging-dose ranges. It has been suggested that 1 in 20 pediatric abdominal CT scans may be inadequate for diagnostic purposes because of radiation-dose-reduction efforts. This will negatively influence the care of some patients because of misguided treatment (7). Importantly, doses cited for nuclear medicine and CT examinations do not even represent patient-specific doses, but rather doses resulting from various models (49). In nuclear medicine, dose optimizers look to lower administered activities, and dosing guidelines for diagnostic-quality images, based on body weight, are available that propose to have a positive impact on uniform pediatric dosing. But approaches based solely on administered activity are insufficient because they ignore interpatient biokinetics, which are highly variable, significantly affecting dose estimates and image quality. For example, in some patients, radiopharmaceutical clearance is quicker than average, and the result may be a suboptimal image resulting in lower counts and increased image noise from inappropriately reduced administered activity. Recently, a methodology incorporating adjustment for body morphometry, use of age-specific biokinetics, and more detailed phantom modeling has been described as a first step in reducing pediatric absorbed dose while maintaining image quality; but although image quality has objective, measurable properties, its subjective properties (i.e., radiologist or nuclear physician interpretability) are not easily quantified (50).

The goal of the Image Gently Alliance (51) is to lower the potential risk of CT-caused cancer in children, but this risk is hypothetical, lacking credible evidence. Furthermore, on the basis of the LSS data, children are not more radiosensitive than adults in the imaging dose range. The Alliance mainly addresses pediatric, ALARA-based CT optimization, but without knowledge of actual patient doses and without demonstrated harm at diagnostic imaging doses, this unintentionally misleads and frightens the public.

All medical procedures require justification in the form of medical indication, but radiation exposure levels have no place in that process. There is no excuse for policies and warnings leading to nondiagnostic scans, fear-driven avoidance of medically indicated imaging, or selection of less optimal alternative procedures. The problem is radiophobia, not radiation. Optimization-using doses that are ALARA - is, thus, without justification, only multiplying illnesses, injuries, and deaths. Therefore, the International Commission on Radiological Protection-recommended fundamental principles of radiation protection-justification and optimization-are mutually contradictory and without merit for radiologic imaging and other sources of low-dose radiation exposure as well.

Many grant the absence of low-dose harm yet nevertheless advocate lower imaging dose as a prudent approach; but this conflates actual prudence, restricting medical procedures to those clinically indicated, with the prejudice-based false prudence of 
limiting clinically indicated imaging doses. This unjustified, radiophobia-centered approach falsely vilifies beneficial imaging without confirmatory data and entails extremely harmful consequences. The declaration that the LNTH provides "known" cancer risks due to imaging must stop. The use of the LNTH and the advocacy for ALARA dosing by various groups (e.g., Image Wisely and Image Gently) are misguided and not science- or evidencebased. These groups serve only to frighten rather than to educate, further enhancing the probability of negative outcomes; we therefore recommend that the imaging community come together to decide whether the activities of such groups should be terminated.

\section{MEDICAL IMAGING AND THE FAILURE OF LNTH ORTHODOXY}

Medical imaging is said to carry an iatrogenic risk of cancer from radiation exposure. But credible evidence of cancer risk from imaging, particularly CT and PET/CT scans, is nonexistent; this risk is an imaginary prediction derived from the demonstrably false LNTH. Low-dose radiation from these scans does not cause, but more likely helps prevent, cancer. Actual risk arises from radiophobia through patients' fear-driven imaging avoidance and physician-recommended substitution of alternative procedures. Furthermore, true iatrogenic risk arises not only from such alternative procedures but also from misdiagnoses that are secondary either to patient refusal of medically indicated imaging or to nondiagnostic scans resulting from insufficient exposure. Obtaining correct diagnoses and avoiding riskier alternatives should be paramount; medical imaging is intended to achieve a diagnostic purpose; thus, exposure should not be reduced below the required level to achieve this purpose.

Imaging is a medical procedure that should be governed by the highest, science-based principles and policies (use of proper procedures, appropriately calibrated equipment, etc.). Yet, many believe imaging should be managed by LNTH principles. Herein is the logical and medical fallacy of this conclusion: the LNTH is an invalidated hypothesis, spawning the ALARA principle. It is responsible for misguided concerns promoting dose optimization, leading to risks far greater than even the imaginary low-dose carcinogenic risk it purports to avoid while ignoring the benefits of medical imaging.

With no evidence supporting the LNTH, and much evidence to support hormesis at imaging doses, LNTH advocates are blindly responsible for promoting radiophobia with all its negative consequences. The LNTH and its offspring, ALARA, do not err on the side of caution. Radiophobia can no longer be ignored: proper lowdose radiation exposure has no documented pathway to harm, whereas the LNTH and ALARA most assuredly do.

The only rational and public-health-protective conclusion is that subjecting the life-saving practice of medical imaging to the LNTH is a non sequitur. Medical imaging must no longer suffer in the longstanding thrall of the LNTH. It is incumbent on the medical imaging community to finally and unambiguously denounce the LNTH and, unencumbered by false beliefs, act as advocates for the safety and life-saving benefits of medical imaging.

\section{REFERENCES}

1. Siegel JA, Pennington CW, Sacks B, Welsh JS. The birth of the illegitimate linear no-threshold model: an invalid paradigm for estimating risk following low-dose radiation exposure. Am J Clin Oncol. November 3, 2015 [Epub ahead of print].

2. Siegel JA, Stabin MG. RADAR commentary: use of linear no-threshold hypothesis in radiation protection regulation in the United States. Health Phys. 2012;102:90-99.

3. Siegel JA, Welsh JS. Does imaging technology cause cancer? Debunking the linear no-threshold model of radiation carcinogenesis. Technol Cancer Res Treat. 2016;15:249-256.
4. Dobrzyński L, Fornalski KW, Feinendegen LE. Cancer mortality among people living in areas with various levels of natural background radiation. Dose Response. 2015;13:1559325815592391.

5. Dauer LT, Brooks AL, Hoel DG, et al. Review and evaluation of updated research on the health effects associated with low-dose ionising radiation. Radiat Prot Dosimetry. 2010;140:103-136.

6. Balter S, Zanzonico P, Reiss GR, Moses JW. Radiation is not the only risk. AJR. 2011;196:762-767.

7. Brody AS, Guillerman RP. Don't let radiation scare trump patient care: 10 ways you can harm your patients by fear of radiation-induced cancer from diagnostic imaging. Thorax. 2014;69:782-784.

8. Zanzonico PB. The neglected side of the coin: quantitative benefit-risk analyses in medical imaging. Health Phys. 2016;110:301-304.

9. Cohen MD. CT radiation dose reduction: can we do harm by doing good? Pediatr Radiol. 2012;42:397-398.

10. Cohen MD. ALARA, Image Gently and CT-induced cancer. Pediatr Radiol. 2015;45:465-470.

11. National Research Council of the National Academies. Health Risks from Exposure to Low Levels of Ionizing Radiation: BEIR VII Phase 2. Washington, DC: The National Academies Press; 2006.

12. Aurengo A, Averbeck D, Bonnin A, et al. Dose Effect Relationships and Estimation of the Carcinogenic Effects of Low Doses of Ionizing Radiation. Paris, France: Académie des Sciences-Académie nationale de Médecine; March 2005.

13. Siegel JA, Sacks B, Stabin MG. LNT 999. Health Phys News. 2015;43:23-24.

14. Ozasa K, Shimizu Y, Suyama A, et al. Studies of the mortality of atomic bomb survivors, report 14, 1950-2003: an overview of cancer and noncancer diseases. Radiat Res. 2012;177:229-243.

15. Ozasa K. Epidemiological research on radiation-induced cancer in atomic bomb survivors. J Radiat Res. 2016;57(suppl 1):i112-i117.

16. Doss M. Linear no-threshold model vs. radiation hormesis. Dose Response. 2013;11:480-497.

17. Sasaki MS, Tachibana A, Takeda S. Cancer risk at low doses of ionizing radiation: artificial neural networks inference from atomic bomb survivors. J Radiat Res (Tokyo). 2014;55:391-406.

18. Boice JD. The Boice report \#40: LNT 101. Health Phys News. 2015;43:25-26.

19. Brooks AL, Dauer LT. Advances in radiation biology: effect on nuclear medicine. Semin Nucl Med. 2014;44:179-186.

20. Luckey TD. Radiation Hormesis. Boca Raton, FL: CRC Press; 1991.

21. Feinendegen LE, Pollycove M, Neumann RD. Low-dose cancer risk modeling must recognize up-regulation of protection. Dose Response. 2009;8:227-252.

22. Löbrich M, Rief N, Kühne M, et al. In vivo formation and repair of DNA doublestrand breaks after computed tomography examinations. Proc Natl Acad Sci USA. 2005;102:8984-8989.

23. Sacks B, Meyerson G, Siegel JA. Epidemiology without biology: false paradigms, unfounded assumptions, and specious statistics in radiation science (with commentaries by Inge Schmitz-Feuerhake and Christopher Busby and a reply by the authors). Biol Theory. 2016;11:69-101.

24. Rothkamm K, Löbrich M. Evidence for a lack of DNA double-strand break repair in human cells exposed to very low x-ray doses. Proc Natl Acad Sci USA. 2003;100:5057-5062.

25. Taylor LS. Some nonscientific influences on radiation protection standards and practice: the 1980 Sievert lecture. Health Phys. 1980;39:851-874.

26. Scott BR, Sanders CL, Mitchel REJ, Boreham DR. CT scans may reduce rather than increase the risk of cancer. J Am Phys Surg. 2008;13:8-11.

27. Billen D. Spontaneous DNA damage and its significance for the "negligible dose" controversy in radiation protection. Radiat Res. 1990;124:242-245.

28. Russo GL, Tedesco I, Russo M, et al. Cellular adaptive response to chronic radiation exposure in interventional cardiologists. Eur Heart J. 2012;33:408414.

29. Gori T, Münzel T. Biological effects of low-dose radiation: of harm and hormesis. Eur Heart J. 2012;33:292-295.

30. Liu SZ. Cancer control related to stimulation of immunity by low-dose radiation. Dose Response. 2006;5:39-47.

31. Chen D, Wei L. Chromosome aberration, cancer mortality and hormetic phenomena among inhabitants in areas of high background radiation in China. $J$ Radiat Res (Tokyo). 1991;2(suppl 2):46-53.

32. Sinclair WK. Science, radiation protection, and the NCRP. Radiation Effects Research Foundation website. http://www.rerf.or.jp/library/update/rerfupda_e/ comment/ncrp.html. Accessed September 6, 2016.

33. Preston DL, Cullings H, Suyama A, et al. Solid cancer incidence in atomic bomb survivors exposed in utero or as young children. J Natl Cancer Inst. 2008; 100:428-436. 
34. United Nations Scientific Committee on the Effects of Atomic Radiation (UNSCEAR). Sources and Effects of Ionizing Radiation: UNSCEAR 1993 Report to the General Assembly, with Scientific Annexes. New York, NY: United Nations; 1993.

35. Siegel JA, Silberstein EB. A closer look at the latest NRC patient release guidance. J Nucl Med. 2008;49(7):17N-20N.

36. Kaushik A, Jaimini A, Tripathi M, et al. Estimation of patient dose in ${ }^{18}$ F-FDG and ${ }^{18}$ F-FDOPA PET/CT examinations. J Cancer Res Ther. 2013;9:477-483.

37. Pearce MS, Salotti J, Little MP, et al. Radiation exposure from CT scans in childhood and subsequent risk of leukaemia and brain tumors: a retrospective cohort study. Lancet. 2012;380:499-505.

38. Mathews JD, Forsythe AV, Brady Z, et al. Cancer risk in 680000 people exposed to computed tomography scans in childhood or adolescence: data linkage study of 11 million Australians. BMJ. 2013;346:f2360.

39. Journy N, Rehel JL, Du Cou Le Pointe H, et al. Are the studies on cancer risk from CT scans biased by indication? Elements of answer from a large-scale cohort study in France. Br J Cancer. 2015;112:185-193.

40. Krille L, Dreger S, Schindel R, et al. Risk of cancer incidence before the age of 15 years after exposure to ionizing radiation from computed tomography: results from a German cohort study. Radiat Environ Biophys. 2015;54:1-12.

41. Fahey FH, Ziniel SI, Manion D, Treves ST. Effects of Image Gently and the North American guidelines: administered activities in children at 13 North American pediatric hospitals. J Nucl Med. 2015;56:962-967.

42. Gelfand MJ, Parisi MT, Treves ST. Pediatric radiopharmaceutical administered doses: 2010 North American consensus guidelines. J Nucl Med. 2011;52:318-322.
43. Goske MJ. Doctor, is a CT scan safe for my child? Br J Radiol. 2014;87:20130517. 44. Zondervan RL, Hahn PF, Sadow CA, Liu B, Lee SI. Body CT scanning in young adults: examination indications, patient outcomes, and risk of radiation-induced cancer. Radiology. 2013;267:460-469.

45. Pandharipande PV, Reisner AT, Binder WD, et al. CT in the emergency department: a real-time study of changes in physician decision making. Radiology. 2016; 278:812-821.

46. Mehanna H, Wong W, McConkey CC, et al. PET-CT surveillance versus neck dissection in advanced head and neck cancer. N Engl J Med. 2016;374:14441454.

47. Brenner D, Elliston CD, Hall EJ, Berdon WE. Estimated risks of radiationinduced fatal cancer from pediatric CT. AJR. 2001;176:289-296.

48. International Commission on Radiological Protection. The 2007 Recommendations of the International Commission on Radiological Protection: ICRP Publication 103. Maryland Heights, MO: Elsevier; 2007.

49. Fahey FH, Treves ST, Adelstein SJ. Minimizing and communicating radiation risk in pediatric nuclear medicine. J Nucl Med. 2011;52:1240-1251.

50. O'Reilly SE, Plyku D, Sgouros G, et al. A risk index for pediatric patients undergoing diagnostic imaging with ${ }^{99 \mathrm{~m}} \mathrm{Tc}$-dimercaptosuccinic acid that accounts for body habitus. Phys Med Biol. 2016;61:2319-2332.

51. Frush DP, Goske MJ. Image Gently: toward optimizing the practice of pediatric CT through resources and dialogue. Pediatr Radiol. 2015;45:471-475. 